\title{
Timing of Delivery of Severe COVID-19 in Pregnancy
}

\author{
David Peleg ${ }^{1}$, Yael Sciaky-Tamir ${ }^{1}$, Steven Warsof ${ }^{2}$, Naama Maimon ${ }^{1}$, Ala Abu Saleh ${ }^{1}$, and \\ Inbar Ben Shachar ${ }^{1}$ \\ ${ }^{1}$ Ziv Medical Center \\ ${ }^{2}$ Eastern Virginia Medical School
}

June 2, 2020

Timing of Delivery of Severe COVID-19 in Pregnancy

David Peleg ${ }^{1}$, Yael Sciaky-Tamir ${ }^{1}$, Steven L. Warsof ${ }^{2}$, Naama Maimon ${ }^{1}$, Ala Abu Saleh ${ }^{3}$, and Inbar Ben Shachar ${ }^{1}$

Department of Obstetrics and Gynecology ${ }^{1}$, and the Intensive Care Unit ${ }^{3}$, Ziv Medical Center, Azrieli, Faculty of Medicine, Bar-Ilan University, Safed Israel, Division of Maternal-Fetal Medicine, Eastern Virginia Medical School, Norfolk, VA, USA ${ }^{2}$,

Correspondence: David Peleg, MD

Ziv Medical Center Rambam Street Safed, Israel 13100 Tel: +972-4-6828959 Fax: +972-4-6828649

Email: david.p@ziv.health.gov.il

Word Count: 1601

Short title: Timing of delivery of SARS-Covid-19

There is no doubt that COVID-19 has had a devastating worldwide affect. As the health care system has scrambled for control, there has been a multitude of reports concerning diagnosis, epidemiology, treatment and prognosis, with new publications appearing each week. Many of these are from personal or area-limited experience. Not infrequent are changing or conflicting results and statistics. The world awaits well-defined and controlled outcome-based research.

One aspect of COVID-19 that remains controversial is the timing of delivery of pregnant women with severe acute respiratory syndrome (SARS-Covid-19) requiring intensive care. Although maternal-fetal medicine obstetricians are an integral part of the multidisciplinary team, there are differing recommendations concerning timing of delivery. The purpose of this report is to propose a guideline for the severely diseased pregnant patient suggesting that pregnant women [?] 32 weeks with SARS-COVID-19 requiring intubation be delivered.

We treated a pregnant woman at 32 weeks' gestation highlighted the dilemma we faced. A 27-year-old, gravida 2, para 1 at $32+5$ weeks' gestational age presented with dry cough, headache, abdominal pain, general weakness of four days' duration. She had a mild fever. Fetal tracing was reactive with no contractions. Chest X-ray revealed an infiltrate in the upper left quadrant and COVID-19 PCR testing was positive. A course of betamethasone for fetal lung maturation was administered and enoxaparin was started. The patient's status deteriorated and medical treatment was initiated with hydroxychloroquine and azithromycin (recommended at the time). On day 4, $\mathrm{O}_{2}$ saturation worsened and she became progressively anxious and restless, with vigorous coughing and tachypnea (up to 50 breaths per minute). A repeat chest X-ray revealed bilateral patchy opacities compatible with Covid-19 ARDS/pneumonia. Fetal monitoring remained 
reassuring. Despite further efforts to improve oxygenation, intubation seemed inevitable. Balancing the risks and benefits, the decision was made to deliver her by cesarean section with general anesthesia and intubation in the operating room, which in retrospect, unnecessarily exposed the staff to additional time of the patient's uncontrollable coughing, irritability and cognitive disturbance due to hypoxia. A $2200 \mathrm{~g}$ male baby was delivered with Apgars of 2 and 7 (cord arterial pH $7.18 \mathrm{BE}=-5.9$ ). In the NICU the baby required 4 days of nasal CPAP due to mild respiratory distress. Covid-19 was negative twice. The mother remained intubated for an additional 5 days with gradual improvement in her status. She was extubated at that time and discharged from the hospital on day 8 post-operation. The baby was discharge in good condition 15 days after birth.

We propose that pregnant women [?] 32 weeks' gestation with SARS-COVID-19 requiring mechanical ventilation (critical disease) be delivered (Figure 1) without delay. Illness from SARS-COVID-19 proceeds along a continuum from mild to severe disease. Progression may be rapid. There is considerable maternal and fetal morbidity and mortality with critical disease. Delay in delivery after 32 weeks exposes the mother and fetus to continued risks, while delivery may improve the maternal condition. Prior to 32 weeks, delivery should be individualized and discussed in a team setting, taking into account maternal or fetal status (1).

The risk of death with severe disease is substantial. The reported risk of maternal death during the three corona virus pandemics (SARS, MERS, Covid-19) was 12.3\% (2). In a USA nationwide study between 2006 - 2012, the overall risk of maternal death from ARDS in the USA was 9\% (3). Age specific mortality from SARS-COVID-19 is at this time difficult to determine from the literature. In the age group $20-49,2.4 \%$ (6 of 255) of female patients with severe disease died in New York (4). In China, the case fatality ratio in the age group $20-49$ was $0.14 \%$ (both sexes) (5). In Iran, of 9 reported cases of pregnant women with severe COVID-19 disease, 7 died (77.8\%) (6)

All pregnant women with COVID-19 infections should be triaged to one of three classes: mild moderate or severe (7). Mild signs and symptoms (mild disease) can be managed in the outpatient setting (Figure 1). Some will have worsening signs symptoms (dyspnea, decreased oxygen saturation). Patients with moderate disease should be hospitalized for continued evaluation and treatment. Pregnant women with severe disease will have marked tachypnea, oxygen saturation [?] 93\%, partial pressure of arterial $\mathrm{O}_{2}$ to inspired oxygen fraction $<300$ ), and infiltrates of at least $50 \%$ of lung fields $(7)$. These women require intensive care.

At least three-quarters of patients with SARS-COVID-19 will require mechanical ventilation. Known risk factors for severe disease include older age ( $>65$ years), chronic lung disease, cardiovascular disease, diabetes mellitus, obesity, immunocompromised, renal disease, and liver disease (7). It is unknown whether pregnancy, while adding an extra burden to the sick mother, contributes to maternal deterioration. It should be remembered that pregnant women during the SARS-CoV 2012 epidemic and the H1N1 2009 pandemic had higher morbidity and mortality than non-pregnant women (8). Data for COVID-19 is incomplete.

The changes in maternal physiology would be expected to negatively affect the prognosis of women with severe respiratory disease requiring mechanical ventilation. For example, pregnancy causes an increase in cardiac output up to $40 \%$. Blood pressure is lower due to a decrease in systemic vascular resistance, and serum albumin and colloid osmotic pressure are decreased, placing the pregnant women at risk for thirdspacing of fluid, volume overload, and pulmonary edema. Respiratory changes in pregnancy include an increase in minute ventilation with a compensated respiratory alkalosis.

For these and other reasons, mechanical ventilation of the pregnant patient requires special consideration. Pregnant women who develop respiratory failure and require mechanical ventilation should be ventilated to a target $\mathrm{PaCO}_{2}$ between $28-30 \mathrm{~mm} \mathrm{Hg}$ to avoid alterations in uterine blood flow and fetal oxygen delivery. Functional residual capacity is decreased $10-25 \%$ as term approaches, and places an extra burden on a women requiring mechanical ventilation due to hypoxemic respiratory failure. It is desirable to maintain low tidal volume ventilation, higher peak inspiratory pressure, and positive end expiratory pressure. Hyperventilation should be avoided. To guarantee sufficient fetal oxygenation, maternal $\mathrm{PaO}_{2}$ should not be below $70 \mathrm{~mm}$ Hg. Targeting lower ventilation pressures may not be feasible in pregnant patients where intra-abdominal 
pressure would be physiologically increased. Certainly pregnancy adds an unquantified burden to the medical team responsible for caring for the severely ill patient at significant risk of death.

There exists a lack of evidence-based guidelines concerning mechanical ventilation of pregnant patients with ARDS, as well as the effect of delivery on maternal and fetal wellbeing. Of 29 pregnant patients, 10 were delivered while mechanically ventilated. Following delivery, 3 had a $50 \%$ decrease in oxygenation index and 5 had a greater than $50 \%$ increase in lung compliance (9). Extracorporeal membrane oxygenation has been shown to be of some benefit in pregnant women with critical ARDS. One study showed a $77 \%$ maternal and a $56 \%$ fetal survival rate (10). Although data are equivocal, delivery can be expected to improve fetal wellbeing (9). However, other have argued that delivery should not be performed solely to improve maternal oxygenation.

The prolonged hypoxemia associated with severe respiratory disease would be expected to have a detrimental effect on the fetus. Maternal hypoxia reduces placental blood flow and fetal oxygenation due to vasoconstriction. Maternal hypercapnia may produce fetal respiratory acidosis (9). Along with growth restriction, chronic hypoxia has been associated with fetal brain injury (11). Long term effects of hypoxia include increased risks of cardiovascular, metabolic and renal disease. Medications used for sedation may adversely affect the fetus. The pooled percentage of perinatal death among pregnancies with SARS-Covid-19 was $7 \%$ (2). Of 9 pregnant women with SARS-COVID19 in the 2 nd and $3^{\text {rd }}$ trimesters who succumbed to the disease, 4 (44\%) had intrauterine fetal demise not related to prematurity (6).

The modality most relied upon to determine fetal status is the cardiotocograph (CTG). Other modalities, such as biophysical profile and doppler studies that are useful with growth restriction, may be impractical and have not been thoroughly evaluated in these situations. A normal appearing CTG tracing may give the obstetrician a false sense of security in the severely sick, anesthetized and intubated woman, and waiting for classical CTG changes of fetal distress may be unsafe.

Although vaginal delivery would seem preferable and may be considered in the stable patient, induction of labor in the severely sick pregnant woman is most often unreasonable. Of the 9 pregnant women dying from SARS-COVID19, 6 underwent cesarean, 1 had a vaginal birth, and 2 died undelivered (6). Of 20 pregnant women critically ill with Covid-19 admitted at 30.6 weeks mean gestational age, 16 (94\%) were delivered by cesarean at 31.9 weeks mean gestation (5 days after hospitalization) (12). A controlled delivery by cesarean is the treatment of choice in these women. Regional anesthesia may be attempted, however, these women are very sick and on the verge of requiring mechanical ventilation; general anesthesia with intubation seems the more logical approach. Special efforts are needed to minimize staff exposure.

We believe that the decision to delay delivery after 32 weeks in the SARS-COVID-19 patients on the verge of becoming critically ill has the potential to cause more harm than benefit. Before 32 weeks, the decision to delivery may be delayed to benefit the fetus, only if the delay is not expected to worsen the mother's prognosis. Indications for delivery in these situations may include non-reassuring fetal monitoring, intrauterine fetal demise, failing cardiopulmonary status, disseminated intravascular coagulation, and multi-organ failure.

We propose that 32 weeks is a sufficient gestation age to deliver women with SARS-COVID-19 requiring mechanical ventilation since $>98 \%$ intact survival can be expected for these babies. After delivery, concerted efforts for recovery of the mother can be made without fetal concerns.

Disclosure of interests: Each author reports no conflict of interest.

Contributions of authors: Dr. Peleg, Dr. Sciaky-Tamir, Dr. Maimon, Dr. Abu Saleh, and Dr. Ben Shachar contributed to patient care, planning and decision making, design and writing of the manuscript. Dr. Warsof aided in design and writing of the manuscript.

Ethics approval: The present work was deemed exempt from obtaining Ethics committee approval.

Funding: There were no sources of funding support

References 
1. Management considerations for pregnant patients with COVID-19. Society for MaternalFetal Medicine. https://s3.amazonaws.com/cdn.smfm.org/media/2336/SMFM_COVID_Management_of_COVID_pos_preg_patients_4-30-20_final.pdf.

2. Di Mascio D, Khalil A, Saccone G, et al. Outcome of Coronavirus spectrum infections (SARS, MERS, COVID 1 -19) during pregnancy: a systematic review and meta-analysis. Am J of Obstet Gynecol MFM (2020), doi:https://doi.org/10.1016/j.ajogmf.2020.100107.

3. Rush B, Martinka P, Kilb B, McDermid RC, Boyd JH, Celi LA. Acute Respiratory Distress Syndrome in Pregnant Women. Obstet Gynecol 2017;129:530-5.

4. Richardson S, Hirsch JS, Narasimhan M, Crawford JM, McGinn T, Davidson KW; and the Northwell COVID-19 Research Consortium. Presenting characteristics, comorbidities, and outcomes among 5700 patients hospitalized with COVID-19 in the New York City area. JAMA. 2020 Apr 22. doi: 10.1001/jama.2020.6775.

5. Verity R, Okell LC, Dorigatti I, et al. Estimates of the severity of coronavirus disease 2019: a model-based analysis. Lancet Infect Dis. 2020 Mar 30. pii: S1473-3099(20)30243-7. doi: 10.1016/S1473-3099(20)30243-7.

6. Hantoushzadeh S, Shamshirsaz AA, Aleyasin A, et al. Maternal death due to COVID-19 disease. Am J Obstet Gynecol 2020 https://doi.org/10.1016/j.ajog.2020.04.030.

7. Gandhi RT, Lynch JB, del Rio C. Mild or moderate Covid-19. N Engl J Med 2020 Apr 24. doi: 10.

8. Lam CM, Wong SF, Leung TN, et al. A case-controlled study comparing clinical course and outcomes of pregnant and non-pregnant women with severe acute respiratory syndrome. BJOG 2004;111:771-4.

9. Lapinsky SE, Rojas-Suarez JA, Crozier TM, et al. Mechanical ventilation in critically-ill pregnant women: a case series. Int J Obstet Anesth 2015;24:323-8.

10. Moore SA, Dietl CA, Coleman DM. Extracorporeal life support during pregnancy. J Thorac Cardiovasc Surg 2016;151:1154-60.

11. Dong Y, Yu Z, Sun Y, et al. Chronic fetal hypoxia produces selective brain injury associated with altered nitric oxide synthases. Am J Obstet Gynecol 2011;204:254.e16-28.

12. Pierce-Williams RAM, Burd J, Felder L, et al. Clinical course of severe and critical COVID-19 in hospitalized pregnancies: a US cohort study. Am J Obstet Gynecol MFM (2020), doi:https://doi.org/10.1016/j.ajogmf.2020.100134.

Legend to Figure 1

Delivery of severe Covid-19 in pregnancy 


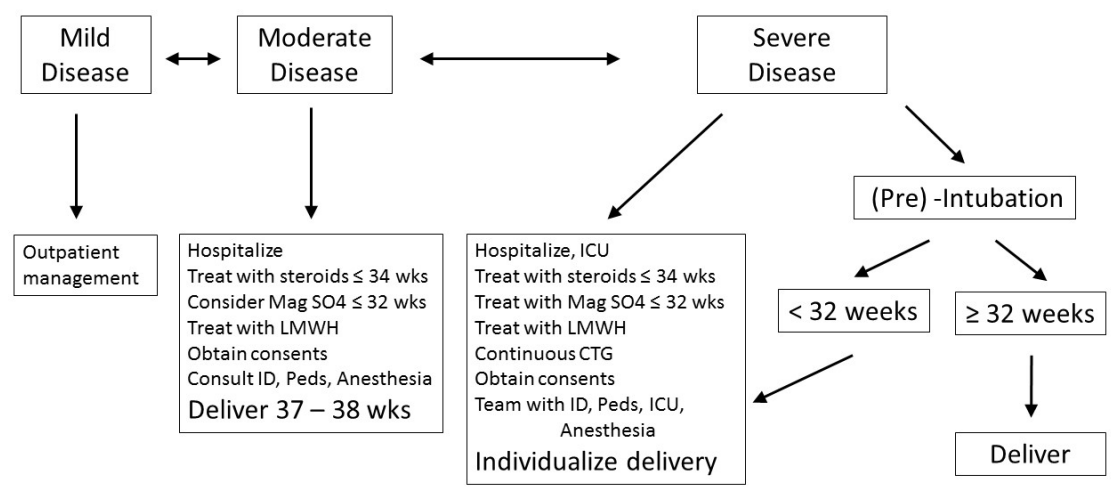

